\title{
Haemoglobinopathies care and cure: Have we reached the end?
}

\author{
John Porter \\ Professor of Haematology, University College London and Joint Red Cell Unit, UCLH and \\ Whittington Hospitals, London, UK
}

Recent years have seen accelerating advances in the treatment, monitoring and potential cures for haemoglobin disorders, as the interaction between basic science, pharmaceutical research, and practical medicine intensifies. In order to appreciate how close to the end we may have reached, it is helpful to consider the journey thus far.

For thalassaemia syndromes, advances with non-curative treatment began with the establishment of the principles of blood transfusion and their application in the 1950s both to treat anaemia and suppress erythropoietic expansion. The consequences of transfusional iron overload soon became a problem however, with patients dying in their late teens and early 20 s, typically with cardiomyopathy. The introduction of desferrioxamine infusions in the 1970s, led to gradual improvement in outcome and the subsequent introduction and licensing of orally active chelation has contributed to improved treatment adherence and improved survival. Morbidity from iron overload, particularly hypogonadotrophic hypogonadism remains a serious issue and emergence of new pathologies in older patients, such as in the liver, is a cause for concern. Curative treatment with allogeneic stem cell transplantation was introduced in the 1980 s but is only available to a minority of patients and is still associated with significant morbidity and mortality. Novel approaches aimed at decreasing transfusion requirements by improving the efficiency of erythropoiesis, such as with activin receptor traps, may prove useful in both transfusion dependent and non-transfusion dependent thalassaemias. Gene therapy is now a reality for a small number of patients and has the potential for application to many patients in whom allogeneic transplant was precluded by lack of a suitable donors, or was too risky for other reasons such increasing as age.

For sickle cell disorders, advances have included the setting up of specialist clinics, pneumococcal septicaemia prevention programs, the application of blood transfusion for the prevention and treatment of disease complications, the use of hydroxyurea for prevention of painful crises and chest syndrome and allogeneic transplantation for carefully selected patients. New pharmacological agents with novel mechanisms of action are being evaluated at a hitherto unprecidented rate. However thus far, the impact of these advances on survival and quality of life in patients as a whole often lags somewhat behind those of thalassaemia. Disease prevention is a key element to management of both sickle and thalassaemia syndromes but implementation has been highly variable both geographically and even between these conditions at a local level. Prevention of births with sickle cell disorders have been less effective than for thalassaemia, even in countries such as the UK which share the same prevention programs for these conditions. The perceived variability and unpredictability of sickle cell disease is part of the reason for this: if all patients had a uniformly fatal outcome without transfusion, blood transfusion would be more uniformly applied, as with TDT, from an early age with perhaps better overall quality of life and survival.

In order approach "the end," advances in therapy will need to be more affordable and deliverable to populations where the conditions are most prevalent. It is anticipated in the next decade that blood transfusion safety will improve, the cost of chelation will fall, the safety and applicability of allogeneic stem cell transplantation with increase, and with further scientific advances such as CRISPR technology, the cost of gene therapy fall. In the meantime, to paraphrase a well-known quotation, "this is not the end, it is not even at the beginning of the end, but it is perhaps the end of the beginning".
Correspondence: John Porter, Haematology, University College London and Joint Red Cell Unit, UCLH and Whittington Hospitals, London, UK

This work is licensed under a Creative Commons Attribution 4.0 License (by-nc 4.0).

CC Copyright J. Porter, 2018

Licensee PAGEPress, Italy

Thalassemia Reports 2018; 8:7470

doi:10.4081/thal.2018.7470 